\title{
Traduire
}

Une eutre perspective sur $r$ tatadciction

Revue française de la traduction

$226 \mid 2012$

Face au miroir

\section{Réflexion sur la consécration par la traduction}

(à partir de l'exemple d'auteurs polonais contemporains et de leurs traducteurs)

\section{Monika Grabowska}

\section{(2) OpenEdition}

12 Journals

Édition électronique

URL : http://journals.openedition.org/traduire/148

DOI : $10.4000 /$ traduire. 148

ISSN : 2272-9992

Éditeur

Société française des traducteurs

Édition imprimée

Date de publication : 1 janvier 2012

Pagination : $59-71$

ISBN : 039-773X

ISSN : 0395-773X

Référence électronique

Monika Grabowska, «Réflexion sur la consécration par la traduction », Traduire [En ligne], 226 | 2012, mis en ligne le 03 février 2014, consulté le 09 octobre 2020. URL : http://journals.openedition.org/ traduire/148; DOI : https://doi.org/10.4000/traduire.148 


\title{
Réflexion sur la consécration par la traduction
}

\author{
(à partir de l'exemple d'auteurs polonais contemporains et de leurs \\ traducteurs)
}

Monika Grabowska

\section{Remarques préliminaires}

1 Le présent article est la continuation d'un projet que nous avons conçu en 2010 et dont le but était de contribuer à la réflexion sur la (les) figure(s) du traducteur aux yeux de l'auteur. Nous sommes alors partis du principe que

\begin{abstract}
le traducteur "avait» ses auteurs, et que ceux-ci, notamment dès qu'ils commençaient à être traduits, se créaient une vision particulière du travail du traducteur. Autrement dit, nous nous sommes proposé d'examiner la relation entre deux personnes, le traducteur et l'auteur, et son impact sur la représentation que ce dernier se fait de l'activité professionnelle du premier ${ }^{1}$.
\end{abstract}

2 Nous avons donc décidé d'interviewer quelques écrivains et dramaturges polonais sur leurs relations avec les traducteurs; cela nous a permis d'arriver à la conclusion que l'image du traducteur aux yeux de l'auteur s'inscrivait tantôt dans le plan des relations interpersonnelles, impliquant la reconnaissance mutuelle de la valeur du texte d'auteur ainsi que celle du talent du traducteur pour le transmettre dans une autre langue/ culture, tantôt sur le plan des affaires économiques, résultant d'un intérêt commun (l'un n'excluant aucunement l'autre). Il semble en tout cas que Françoise BarretDucrocq (1992 : 207) ait raison en affirmant que « les histoires de traductions heureuses (...) sont clairement celles où traducteur et auteur ont su établir des liens très forts, qu'ils soient amicaux, passionnels ou intellectuels ». En effet, il résulte de notre enquête que :

l'angle d'approche des relations auteur-traducteur dominant pour les contemporains qui ont eu la chance de se connaître est fréquemment, du point de vue de l'auteur, celui du lien psychologique plus ou moins fort, mais n'exclut pas la vision du traducteur en tant qu'instance énonciative (voix de l'auteur décalée), 
juridique (portant la responsabilité de sa qualité), économique (jouissant des droits de traducteur, quelque modestes qu'ils soient). (Grabowska 2012, à paraître)

Il convient aussi de souligner que tous les auteurs interviewés sont unanimes sur un point : la joie d'accéder au rang d'auteur traduit. Les interviews avec Marek Krajewski, Olga Tokarczuk, Przemysław Wojcieszek, Małgorzata Sikorska-Miszczuk et Zyta Rudzka ont prouvé le prestige dont jouit le traducteur dans la culture polonaise. Il se base sur le respect de ses capacités intellectuelles et du difficile travail auquel il doit s'adonner, et se manifeste à travers quelques nouvelles métaphores à ajouter au répertoire déjà connu ${ }^{2}$, notamment : le traducteur est un " confesseur ", un " critique-contrôleur ", un "promoteur ", un "seigneur et maitre " et finalement un "consacrant ", métaphore empruntée à Casanova 2002 pour la traduction littéraire. Puisque la figure du consacrant constitue le point de départ de cet article, elle mérite une explication plus détaillée.

4 En effet, Casanova conteste «la représentation du monde littéraire selon laquelle on aurait affaire à une juxtaposition d'univers autosuffisants, fermés et irréductibles les uns aux autres, et de langues égales, séparées et autarciques », bien au contraire :

Les inégalités et les hiérarchies, tant littéraires que linguistiques, qui ordonnent le champ littéraire mondial font apparaître une autre économie des échanges linguistiques : loin d'être l'échange horizontal ou le transfert pacifié souvent décrit, la traduction ne peut être comprise, au contraire, que comme un « échange inégal » se produisant dans un univers fortement hiérarchisé. Du même coup, elle peut être décrite comme l'une des formes spécifiques du rapport de domination qui s'exerce dans le champ littéraire international ; et aussi, de ce fait, comme un enjeu essentiel des luttes pour la légitimité qui se livrent dans cet univers, c'est-à-dire comme l'une des voies principales de consécration ${ }^{3}$ des auteurs et des textes. (Casanova $2002: 7$ )

La chercheuse divise par conséquent les langues du monde en deux catégories : langues dominantes et langues dominées. Les premières, « du fait de leur prestige spécifique, de leur ancienneté, du nombre de textes déclarés universels écrits dans ces langues, sont dotées d'un volume important de capital littéraire ». Les secondes peuvent, à leur tour, être classées en quatre sous-catégories : les langues orales ou celles dont l'écriture est récente (l'exemple donné par l'auteur étant celui de certaines langues africaines), les langues qui ont émergé au moment d'une indépendance relativement récente pour obtenir le statut de langue nationale (l'hébreu, le néo-norvégien...), les langues de tradition ancienne, mais liées à de petits pays (le grec, le danois), et enfin les langues de grande diffusion (l'arabe, le chinois) mais peu connues sur le marché littéraire international. Le polonais fera sans doute partie du troisième groupe : il est ancien (il date du Moyen-Âge), mais a peu de locuteurs (environ 40 millions, à mettre en regard avec le nombre approximatif de 160-200 millions de francophones), est peu pratiqué par les polyglottes et peu connu en dehors des frontières nationales.

Casanova évoque à titre d'exemple «le processus de consécration» de l'écrivain polonais Witold Gombrowicz, commencé lors de sa traduction en français par Constantin Jelenski, renforcé par la publication du roman Ferdydurke par l'éditeur Maurice Nadeau et achevé par les commentaires et analyses de l'écrivain Dominique de Roux. Le sentiment d'une semblable consécration émerge des propos de Zyta Rudzka, romancière et dramaturge que nous avons interviewée. Elle estime que le fait d'avoir été traduite par des personnes de l'envergure d'Esther Kinsky, Ursula Kiermeier ou Martin Pollack est un passeport pour un public allemand élitiste. Casanova n'oublie toutefois pas de remarquer que la situation peut s'inverser : un écrivain reconnu est 
susceptible de consacrer le traducteur. En effet, les auteurs interviewés dans nos enquêtes ont souvent souligné les noms des écrivains prestigieux qui figurent à l'actif professionnel de «leur» traducteur, les élevant au rang des meilleurs professionnels. Tel est, par exemple, le cas de Doreen Daume que Marek Krajewski n'a pas manqué de présenter comme traductrice de Bruno Schulz. Ainsi, la gloire de l'auteur des Boutiques de cannelle (par exemple) passe sur son traducteur, qui, à son tour, valorise un nouvel auteur lorsqu'il se décide à le traduire...

7 Après avoir confirmé le statut consacrant du traducteur d'œuvres polonaises vers les langues dominantes grâce aux témoignages des écrivains contemporains, nous avons entrepris de confronter leur opinion à celle de leurs traducteurs, qui ont été interviewés à leur tour par courrier électronique. Nous avons contacté, par ordre alphabétique : Bożena Antoniak (traductrice du polonais en ukrainien), Irina Kisielowa (en russe), Antonia Lloyd-Jones (en anglais), Paulina Schulz (en allemand), Włodzimierz Szymaniak $^{4}$ (en portugais), Andreas Volk (en allemand). Nous devons avouer avec regret que le questionnaire destiné aux traducteurs a été élaboré en parallèle avec le questionnaire pour les auteurs, et que les deux ont été envoyés simultanément aux deux groupes-cibles. Ainsi, celui des traducteurs ne tient pas compte des conclusions tirées de l'analyse des réponses des auteurs, ni pour les étayer ni pour les réfuter. Nous trouvons toutefois que, quelque psychologisante qu'elle puisse paraître, notre enquête effectuée auprès des traducteurs est aussi révélatrice de la relation à la fois vitale et symbolique qui relie le traducteur et l'auteur dans un projet commun, en rendant le premier consacrant du deuxième.

8 Pour le présent article, nous avons choisi d'analyser les réponses à huit questions de l'enquête. Les questions seront présentées ci-dessous dans la traduction française et suivies d'une brève synthèse commentée des réponses des traducteurs interviewés. Nous nous référerons aussi à quelques interviews avec les traducteurs du polonais en allemand consultées sur le site du Goethe Institut.

\section{Pourquoi avez-vous choisi de traduire l'œuvre de l'auteur $X$ ? \\ En général, quels sont les critères de sélection des textes littéraires que vous vous décidez à traduire?}

Cette question suppose que le traducteur joue un rôle d'instance de marketing, ce qui n'est pas toujours conforme à la réalité, mais concerne justement le cas des traducteurs-éditeurs que sont par exemple Bożena Antoniak et son mari Anatoli Ivchenko. En 2010, ils ont fondé à Lvov la maison d'édition Urbino se spécialisant dans la traduction des auteurs polonais contemporains en ukrainien. Ainsi, Antoniak jouit du rare privilège de pouvoir jeter son dévolu sur tel ou tel auteur susceptible d'intéresser le public ukrainien. Pourtant, s'agit-il toujours d'une consécration? Les deux langues, le polonais et l'ukrainien, se situent dans le même camp des langues dominées, mais avec une différence de statut, la langue ukrainienne n'étant que depuis très récemment la langue d'un pays souverain, et l'Ukraine n'étant pas membre de l'Union européenne (l'accession à l'UE peut être vue, dans ce contexte, comme une promotion). À l'époque du communisme, le polonais était pour maints Ukrainiens une langue véhiculaire grâce à laquelle ils pouvaient accéder aux œuvres des auteurs interdits en URSS mais traduits en polonais (entre autres grâce aux maisons d'édition polonaises à l'étranger, dont les publications étaient introduites clandestinement dans le pays). Ainsi, en établissant une hiérarchie du pouvoir dans le champ des langues dominées, si hiérarchie du pouvoir il y a, il serait difficile de situer l'ukrainien au-dessus du polonais. Ceci dit, nous sommes d'avis qu'il n'est pas 
judicieux de rejeter complètement la métaphore de la consécration puisqu'elle peut être comprise comme le fait, pour une œuvre, d'avoir été retenue par un traducteur érudit qui l'a jugée digne d'être intro- duite dans une autre culture. Ainsi, le traducteur, par son statut de sage électeur, lui confère un caractère universel, supranational, sublime...

Les réponses des autres traducteurs à la première question insistent sur le fait qu'ils sont obligés de respecter le choix des éditeurs, mais qu'il leur arrive de refuser la traduction d'un texte qu'ils n'ont pas apprécié ou, au contraire, de convaincre les éditeurs de diffuser des œuvres qui ont attiré leur attention. Cette attitude de combattant (visible dans les métaphores utilisées par les interviewés, comme celle de «lutter» pour gagner l'intérêt des éditions pour telle ou telle œuvre à traduire) résulte sans doute, si nous pouvons nous permettre une interprétation psychologisante, d'un sentiment de mission liée à une fascination de la culture source (liée à la langue source). Ainsi, sur le site du Goethe Institut, Esther Kinsky confie s'être fortement impliquée dans la recherche d'un éditeur pour une nouvelle traduction de Lalka (trad. fr. La poupée) de Bolesław Prus, chef-d'œuvre du roman réaliste polonais publié en 1890, mine de renseignements sur l'identité polonaise et référence incontournable de la littérature polonaise moderne. De la même manière, Martin Pollack (cf. sitographie) a d'ores et déjà entrepris de multiples démarches pour aboutir à la parution sur le marché allemand de Czarny ogród, un romanreportage de Małgorzata Szejnert. Cette tâche lui paraît d'ailleurs tellement ingrate qu'il n'hésite pas à se comparer à un démarcheur ou à un commis-voyageur. Tous les traducteurs sont d'avis que leur travail consisterait dans l'idéal à traduire exclusivement des textes qu'ils auraient sélectionnés eux-mêmes pour leur valeur littéraire ou par sympathie personnelle, et pas sur des critères de marketing.

La culture source peut (pour W. Szymaniak et P. Schulz) ou non (B. Antoniak, A. Lloyd-Jones, I. Kisielowa, A. Volk) être la culture maternelle du traducteur, mais elle est toujours chez les traducteurs interviewés une culture affective. Autrement dit, aucun d'entre eux n'a été réduit à devenir traducteur à partir de ou vers une langue qui suscite chez lui des émotions négatives, des connotations déplaisantes ou douloureuses. Leur mission de créer des connivences entre deux cultures est souvent complètement gratuite, basée sur un altruisme plus que sur un calcul économique ${ }^{5}$, et fait des traducteurs des médiateurs interculturels stricto sensu. En sont-ils conscients?

\section{En traduisant, avez-vous l'impression de jouer un rôle de médiateur culturel ?}

Un traducteur du polonais en allemand, Martin Pollack, souligne dans une interview publiée sur le site du Goethe Institut que la médiation interculturelle au sens large fait partie de la profession du traducteur. Cette conviction est partagée par Paulina Schulz et Andreas Volk qui énumèrent les nombreuses actions interculturelles auxquelles ils collaborent, voire qu'ils ont inspirées. Il n'est peut-être pas sans importance de préciser que tous les trois sont liés au marché germanophone (en Allemagne et en Autriche) et assument donc une mission particulière d'effacement des stéréotypes nationaux et d'atténuation des tensions polono-allemandes. De telles tensions ont récemment été créées autour de la Fédération des expulsés d'Erika Steinbach qui a justement rendu problématique l'édition de Czarny ogród de Szejnert, car ce livre est une contribution à l'histoire de la Haute-Silésie qui, aux dires des 
éditeurs (cf. l'interview en ligne avec M. Pollack), risquerait de leur valoir une étiquette de maison d'édition des Allemands expulsés de Silésie après la guerre.

Les réponses des autres traducteurs ayant été succinctes (« oui », «bien sûr »), leur mutisme peut être interprété comme un manque d'envie de se répandre sur cette lapalissade dans le contexte polono-anglais, polono-portugais ou encore polonorusse. Cependant, la représentante de la culture ukrainienne évoque son rôle non de médiateur, mais d'explorateur de terres inconnues dont elle importe des fragments chez elle. La médiation transparaît quand même dans l'allusion à la connaissance des cultures que ce rôle favorise - dans ce cas précis, des cultures géographiquement proches, puisque voisines, mais souvent complètement méconnues puisque ne faisant pas partie de l'univers convoité des cultures dominantes. Ainsi, Bożena Antoniak s'estime fière et privilégiée d'être celle qui a introduit Tadeusz Konwicki en Ukraine. Il faut d'ailleurs ajouter qu'elle maîtrise aussi les langues serbo-croate et sorabe, qualifiant cette dernière de complètement ignorée des Ukrainiens (où ne l'est-elle pas ?...) et dont elle traduit aussi des textes, notamment pour enfants.

Pour conclure cette partie, une autre métaphore peut être évoquée, celle citée par Antonia Lloyd-Jones qui trouve que le traducteur non seulement construit un pont entre les cultures, mais aussi invite les gens à l'emprunter pour s'aventurer sur l'autre rivage. Il est dès lors judicieux de voir comment les traducteurs jugent la part de la création et de la simple reproduction dans cette construction.

3. Trouvez-vous le travail de traducteur aussi créatif que celui de l'écrivain ou du dramaturge?

Cette question nous a paru a priori plus pertinente pour les auteurs. Sans être unanimes sur ce point (puisqu'ils avaient déjà différentes visions de leur propre travail littéraire, se situant eux-mêmes tantôt parmi les artistes, tantôt parmi les artisans), les écrivains ne niaient aucunement la composante créative de la tâche du traducteur. Quant aux traducteurs, certains sont intransigeants sur ce point: la création fait partie intégrante de leur métier, parfois même dans une plus grande mesure que chez l'auteur (!). Andreas Volk (en ligne) cite à ce propos l'argument d'un texte contemporain polonais pour lequel il est absolument convaincu qu'un auteur allemand de la même génération ne l'aurait jamais écrit de la même façon. Il s'agit en l'occurrence d'un recours fréquent et spontané des jeunes auteurs polonais au style et aux métaphores religieux, surtout bibliques, comme dans le cas d'un drame de Mariusz Bieliński ( $\mathrm{Nad}$ ). Le rôle du traducteur est alors de s'ingénier à inventer une stratégie pour rendre le texte acceptable en Allemagne sans défigurer le message qu'il véhicule. Cette idée trouve un prolongement dans la réponse d'Irina Kisielowa, selon laquelle le traducteur doit trouver un style vraisemblable qui permettra à l'idée contenue dans le texte d'atteindre le public étranger.

D'autres traducteurs présentent des opinions plus modérées : le fait d'accomplir une traduction ne fait pas pour autant appartenir le livre au traducteur, il reste dans le domaine des biens intellectuels de l'auteur. La valeur sur laquelle les traducteurs appuient leur réponse est celle de la liberté, intrinsèquement liée à la création. C'est elle qui divise le monde des créateurs en absolus et partiels. La question qui se pose alors est celle de savoir si la liberté partielle est toujours une liberté... Dans l'hypothèse où il n'existerait pas une seule traduction valable et idéale, mais une multitude d'options, nous souscrivons plutôt à l'opinion de la majorité des traducteurs interviewés selon laquelle respecter un modèle de référence n'équivaut 
pas à devenir esclave. Si c'était le cas, il faudrait contester aussi la valeur littéraire des parodies, des scénarios créés à partir d'un texte littéraire ou des poèmes respectant fidèlement les contraintes de genre.

L'un des traducteurs résume ainsi la réponse à la question sur l'apport créatif du travail du traducteur : il est bien créatif, mais autrement que l'auteur, puisqu'il ne crée pas ex nihilo; en revanche, il a beaucoup de limites à respecter. Andreas Volk, sur le site du Goethe Institut, ose par conséquent revendiquer pour ses traductions le statut d'œuvres d'art. Ce problème a fait l'objet d'une autre de nos questions.

4. Avez-vous le sentiment qu'en traduisant, vous écrivez votre propre texte et que ce texte, reproduisant théoriquement les traits du texte de l'auteur, est néanmoins lui-même un original, par exemple en raison du style (que le lecteur attribuera d'ailleurs à l'auteur et pas au traducteur...)?

Les traducteurs ne revendiquent pas les droits d'auteur, mais on peut percevoir en filigrane une certaine fierté de savoir trouver un compromis entre le caractère du texte source (qui tout en étant original est aussi discursivement typique), les possibilités de la langue cible (notam- ment pour traduire le fameux « intraduisible ») et les attentes des lecteurs qui ont droit à un texte intelligible (donc typique, se situant dans un genre discursif) et intéressant (original, fidèle au style de l'auteur) en même temps. Bożena Antoniak se révolte par conséquent contre les critiques portant sur la langue de l'auteur dans la version traduite du texte: pour elle, elles sont foncièrement non pertinentes. Il n'y a plus de langue de l'auteur dans un texte traduit. Il y a - selon Volk - une nouvelle qualité. L'auteur de cette nouvelle qualité est le traducteur, mais elle n'aurait jamais été construite sans le texte de départ. Les traducteurs (surtout ceux qui sont eux-mêmes écrivains) avouent être tentés d'améliorer le texte de l'auteur, et donc de commettre une sorte de crime parfait puisque les chances que leur intervention soit révélée sont infinitésimales. En fait, aux dires de ces traducteurs, certains des auteurs finiraient même par avoir peur du traducteur, peur de perdre le contrôle... Il ne s'agit d'ailleurs pas seulement de l'envie $\mathrm{du}$ traducteur d'amender le style de l'auteur, et moins encore de rectifier les inexactitudes qu'il ne manque pas de remarquer (voilà sans doute la raison principale pour laquelle les auteurs voient en leurs traducteurs les critiques les plus sévères). Avant tout, les interviewés conviennent que le traducteur ne devrait surtout pas renchérir sur l'auteur, apporter de nouveaux sens et allusions au texte. Selon Antoniak, le traducteur est bien co-auteur du texte par sa traduction, mais il n'est pas le plus important. Et c'est peut-être en ceci que réside l'un des plus grands choix déontologiques de la profession.

Pour donner néanmoins quelques exemples de corrections «légitimes » effectuées par des traducteurs, nous pouvons citer les romans de Krajewski. Bożena Antoniak confie y avoir détecté une erreur importante liée à une représentation erronée de la topographie de la ville de Lvov. Avec l'auteur, ils ont entrepris de récrire le fragment et ils l'ont aussi signalé aux éditions polonaises. De même, dans le roman Buba de Barbara Kosmowska, la traductrice ukrainienne a remarqué que deux personnages (professeurs de lycée) avaient le même sobriquet «Klinton ». Mais les interventions du traducteur peuvent être plus poussées. La même Bożena Antoniak a, par exemple, constaté que l'un des personnages féminins récurrents dans Buba n'avait pas de prénom. À sa demande, l'auteur a décidé de le baptiser pour les besoins de la traduction ukrainienne. 
Nous avons aussi posé aux traducteurs la question triviale des difficultés auxquelles ils doivent faire face dans l'exercice de leur profession. La liste des problèmes linguistiques semble interminable, mais paradoxalement, elle a surtout été donnée par les auteurs. Maîtres des jeux de mots, ils sont évidemment conscients de l'entreprise périlleuse à laquelle ils condamnent leur traducteur (par exemple, comment rendre en allemand les paroles d'un Polonais qui veut passer pour un Allemand ?). Dans les réponses des traducteurs, il n'est pas tellement question des problèmes de traduction du sens, ni de l'obligation d'explorer un domaine lexical inconnu du traducteur (comme l'astrologie pour les œuvres de Tokarczuk), ni même des difficultés à se documenter (par exemple pour retrouver les noms allemands des anciens restaurants ou hôtels particuliers à Wrocław dans l'entre-deux-guerres). Les traducteurs mettent surtout en valeur le souci de sauvegarder l'originalité du style ou de la tonalité. C'est ici qu'ils avouent avoir le plus de doutes et de tourments intellectuels. Les exemples qu'ils donnent sont multiples. Nous avons déjà cité celui des métaphores bibliques abondantes dans l'œuvre de certains jeunes Polonais et quasi absentes du langage de leurs homologues allemands. Pour l'ukrainien, le problème consisterait à trouver un équivalent plausible du langage familier des milieux urbains polonais compte tenu de sa teneur en vulgarismes. Même un style simple, proche de la tonalité merveilleuse des contes de fées d'olga Tokarczuk, ou le style sobre mais élaboré d'un Ryszard Kapuściński peuvent résister à la traduction. Finalement, les pièces de théâtre de Małgorzata Sikorska-Miszczuk font partie des textes qui défient le traducteur par le goût du pur non-sens, de la paraphrase, du rythme intérieur, tout en gardant une tonalité grotesque et absurde.

Ceci dit, dans l'hypothèse où le texte traduit serait l'œuvre de deux personnes, il serait intéressant de se pencher sur les conséquences d'une telle vision des choses. Le traducteur- consacrant, arrive-t-il toujours à consacrer réellement son œuvre?

5. Comment voyez-vous votre responsabilité dans le succès ou l'échec d'un livre que vous avez traduit?

La réponse à cette question n'est pas évidente puisqu'il y a plusieurs cas de figure. Bożena Antoniak évoque notamment les textes des auteurs dont la valeur a été reconnue dans le pays d'origine (ou d'autres pays) par un accueil enthousiaste du public et de la critique littéraire (comme par exemple El juego del ángel, trad. fr. Le jeu de l'ange de Carlos Ruiz Zafón) et/ou qui sont lauréats de prix prestigieux (comme La vie devant soi de Romain Gary, Goncourt 1975). Pour ces deux titres, la traductrice est impitoyable envers les traducteurs et les rédacteurs ukrainiens : leur incompétence a enterré vives ces œuvres importantes de la littérature moderne puisque les lecteurs ont mis les défaillances du style sur le compte de l'auteur. Elle évoque par ailleurs un autre agent important pour le succès de la traduction : l'éditeur, sans l'intervention publicitaire duquel nombre de bonnes traductions sont malheureusement aussi passées inaperçues en Ukraine. Mais, ce qui est à mettre en valeur dans le contexte de la présente question, c'est le fait que le traducteur est aussi en mesure d'élever un texte quelconque au rang de texte-culte. Irina Kisielowa trouve que tel a été le cas de la traduction de The Catcher in the Rye (trad. fr. L'Attrape-cour) en URSS. Le fait que le texte soit venu de la culture américaine, sans même parler du scandale lié à l'interdiction du roman aux États-Unis, n'est sans doute pas non plus négligeable comme facteur du crédit de confiance chez un public se situant de l'autre côté du rideau de fer. 
Les marchés des ex-républiques soviétiques sont considérés d'une façon générale comme très réceptifs aux nouveautés littéraires. Ce n'est pas tellement le cas du marché britannique où les traductions représenteraient moins de $10 \%$ des publications annuelles. Selon le jugement de Salomon émis par Antonia Lloyd-Jones en réponse à notre question, le traducteur est responsable du succès de la traduction, mais pas de celui du livre, qui résulte de la promotion et des critiques littéraires dans les grands journaux. Toujours est-il que, comme le souligne Włodzimierz Szymaniak, la responsabilité de la traduction est d'autant plus pesante qu'elle perdure, même après la mort du traducteur.

Les traductions de pièces de théâtre constituent un cas à part. Aussi bien les auteurs que les traducteurs affirment que, dans le processus de "consécration", la mise en scène, œuvre commune des réalisateurs et des acteurs, prévaut sur les qualités intrinsèques du texte traduit. Il faudrait d'ailleurs vérifier combien de pièces de théâtre sont de nos jours publiées sans être auparavant mises en scène... En tout cas, les pièces de tous les dramaturges interviewés ont surtout été appréciées à l'étranger en tant que spectacles.

Une autre question que nous avons posée dans l'enquête portait sur les liens personnels entre les deux instances possibles du processus de traduction, l'auteur et le traducteur (où la présence de l'auteur est facultative). Quel peut être l'apport intellectuel de l'auteur à l'entreprise de la traduction? Est-il utile, souhaitable ou insignifiant pour un bon traducteur? Le traducteur a-t-il lui-même besoin d'être (re)connu par l'auteur en tant qu'instance énonciative dans la traduction (on aurait envie de l'appeler alors « interprète », selon l'étymologie du mot)?

6. Quand vous traduisez l'œuvre d'un auteur contemporain vivant, trouvez-vous indispensable de le contacter en personne?

Les réponses des traducteurs évoquent notamment le besoin d'élucider les problèmes ponctuels lors de la traduction. L'aide personnelle de l'auteur pourrait donc faire partie de la boîte à outils du traducteur (nous rejoignons ici la perspective adoptée dans notre article précédent ${ }^{6}$ qui subordonnait l'auteur au traducteur). Ainsi, la figure de l'auteur se dédouble. Si la traduction est une aventure, ne serait-ce qu'intellectuelle, et qu'on reprend les éléments du schéma narratif structuraliste, on pourrait constater que le traducteur est le destinataire (ou le héros), le texte source le destinateur (parfois matérialisé par la personne de l'éditeur), le texte cible l'objet, tandis que l'auteur endosserait, à la fois le rôle de l'opposant (c'est lui qui a créé toutes les difficultés, parsemé d'embûches le chemin de la traduction) et celui de l'adjuvant, détenteur du savoir nécessaire pour déjouer les pièges. C'est l'ultime planche de salut au moment où le traducteur aura épuisé toutes ses ressources personnelles. Andreas Volk avoue y avoir recours notamment dans la traduction de la poésie, pour négocier les pertes. L'auteur joue alors le rôle de conseiller quand il faut choisir quels sens sauvegarder et lesquels sacrifier.

Il est absolument nécessaire de différencier ici le "contact» avec l'auteur (par courriel, mais aussi de vive voix) et la « discussion » qui permet de pénétrer dans la zone individuelle de l'auteur et de mieux comprendre ainsi son émanation sous forme d'un texte d'idées concret. La traductrice-éditrice dit en effet que pouvoir connaître un auteur et discuter avec lui est un privilège dont elle tire parti avec plaisir en se rendant compte qu'elle est admise parfois dans un univers inaccessible 
au commun des mortels. Tel est le cas d'un auteur-légende, senior de la culture polonaise, Tadeusz Konwicki.

Cette discussion peut aussi avoir lieu après la traduction, ce qui est par exemple le cas du prestigieux Congrès des traducteurs organisé par l'Institut du Livre à Cracovie (en 2009, 215 traducteurs de littérature polonaise venus de 56 pays y ont assisté). L'auteur et le traducteur ont aussi parfois l'occasion de promouvoir leur (!) œuvre ensemble, lors de rencontres avec le public, ce qui a fait l'objet de la question suivante.

7. Avez-vous participé à des rencontres de l'auteur avec ses lecteurs étrangers? Si oui, avez-vous reçu des questions de la part du public en tant que traducteur?

Les réponses permettent de constater que la figure du traducteur a une place à part dans la conscience des lecteurs. L'image du traducteur qui sous-tend le choix des questions est celle d'un littéraire. Le public lui pose des questions analogues à celles qui sont devenues stéréo- typées pour les auteurs: raconter sa journée type, présenter ses lectures récentes et ses projets d'avenir... Mais il y aurait aussi des questions plus ciblées sur le métier : quels sont les critères de sélection des textes? Quels sont les écueils de la traduction? À quoi ressemble le cabinet de travail ? Quelles ressources documentaires sont à recommander? Quelles sont les « stratégies » de traduction efficaces ?... Le public s'intéresse aussi à la biographie du traducteur (comment et pourquoi il est devenu traducteur) et aux contacts avec l'auteur (cette question est même posée en présence de celui-ci). On l'interroge aussi sur la relation émotionnelle envers le texte traduit, par exemple quand il doit traduire la description de faits abominables, de crimes et d'atrocités. En terminant notre enquête, nous avons aussi posé ce genre de question.

8. Vous avez traduit plusieurs livres du polonais. Quelles sont les émotions que cette conscience vous inspire?

On répète volontiers que la traduction est une activité ingrate - toujours dans l'ombre, mal rémunérée, sous-estimée... Les auteurs interviewés compatissent généralement au sort de cet écrivain fantôme (même pire, puisque le vrai ghost writer est plutôt bien rétribué). Pourtant, les réponses des traducteurs sont toutes optimistes. Ils évoquent la joie de pouvoir connaître des intellectuels d'envergure, la fierté de contribuer à la publication d'un livre de valeur, la satis- faction de la qualité de la traduction, voire le bonheur tout court de quelqu'un qui a converti sa passion en profession. La fierté se tire aussi des proches (par exemple, d'un ancêtre polonais), et la satisfaction de la collaboration avec un auteur empathique, de la reconnaissance des Polonais, d'une mission bien accomplie... Une nouvelle métaphore du traducteur s'impose ici: celle du joueur (gambler). L'émotion d'un traducteur entendant quelqu'un recommander un livre qu'il a traduit serait celle d'un joueur entrant dans une bonne passe.

\section{Conclusion}

9 Dans Grabowska 2012 (à paraître), nous avons souligné, après Skibińska et Blumczyński 2009, que le traducteur jouissait toujours d'une position prestigieuse dans la culture polonaise. Elle continue d'être le rêve de beaucoup d'étudiants de langues étrangères dans les universités polonaises ${ }^{7}$. Les témoignages des traducteurs consultés les auront sans doute confortés dans leur ambition. Les traducteurs exercent une profession 
noble, certes. Mais n'oublions pas que les «consacrants » font par définition partie d'une caste d'élus qui ont su conduire à la perfection leur compétence au prix d'un travail de bénédictin.

Comme perspective de travail, nous voyons notamment l'élaboration d'un questionnaire visant directement la métaphorisation du traducteur comme «consacrant» et sa soumission à un échantillon de traducteurs des langues «dominées» dans des langues «dominantes» d'une part, et des langues «dominantes » dans des langues «dominées » de l'autre, ainsi qu'à ceux d'une langue "dominante " en une autre langue "dominante » et à ceux d'une langue "dominée " en une autre langue "dominée » (ce que nous avons déjà effleuré avec les traductions du polonais en ukrainien). La confrontation de ces multiples réponses est nécessaire pour confirmer l'existence du « processus de consécration » par le traducteur, dix ans après la parution de l'article de Casanova, alors que la mondialisation a progressé, et peut-être, pour l'opposer à un processus d'« initiation ». Cette initiation concernerait les groupes de lecteurs qui accèdent via la traduction à un fait littéraire prestigieux (tels les poèmes de la poétesse polonaise qui vient de décéder, lauréate du prix Nobel, Wisława Szymborska) ne provenant pas d'une culture vue comme supérieure sur la carte du capital littéraire, mais d'une culture « mineure » (« dominée ») dont la langue est connue de peu de polyglottes.

\section{BIBLIOGRAPHIE}

CASANOVA Pascale, 2002, " Consécration et accumulation de capital littéraire », in Actes de la recherche en sciences sociales 144, p. 7-20.

COOK Jadwiga, 2012, à paraitre, « Figure de rêve ou comment les étudiants se figurent le traducteur », in Romanica Wratislaviensia, p. 59.

D’HULST Lieven, 1993, « Observations sur l'expression figurée en traductologie française (XVIII - $^{-}$ $\mathrm{XIX}^{\mathrm{e}}$ siècles) », in TTR, vol. 6, $\mathrm{n}^{\circ} 1, \mathrm{p}$. 83-111.

BARRET-DUCROCQ Françoise (éd.), 1992, Traduire l'Europe, Paris, Payot.

GRABOWSKA Monika, 2012, à paraître, « Le traducteur vu par son auteur », in Romanica Wratislaviensia, p. 59.

SKIBIŃSKA Elżbieta, 2012, à paraître, « Missionnaire, consacrant, passeur, héraut... ? Figures du traducteur de littérature polonaise en France ", in Romanica Wratislaviensia, p. 59.

SKIBIŃSKA Elżbieta, BLUMCZYŃSKI Piotr, 2009, « Polish metaphorical perceptions of the translator and translations ", in Target $21: 1$, p. 30-57.

\section{Sitographie}

http://www.goethe.de/ins/pl/lp/kul/dup/lit/ueb/pl7196928.htm - interview avec Esther Kinsky, consulté le 13 février 2012. 
http://www.goethe.de/ins/pl/lp/kul/dup/lit/ueb/pl8684113.htm - interview avec Martin Pollack, consulté le 13 février 2012.

http://www.goethe.de/ins/pl/lp/kul/dup/lit/ueb/pl6849474.htm - interview avec Andreas Volk, consulté le 13 février 2012.

\section{NOTES}

1. La communication "Le traducteur vu par son auteur» a été présentée au colloque international Figure(s) du traducteur en octobre 2010 à l'Institut d'Études Romanes de l'Université de Wrocław. L'article paraîtra en 2012 dans la revue Romanica Wratislaviensia ( ${ }^{\circ} 59$ ).

2. À ce propos, voir par ex. D’hulst 1993.

3. Souligné par Monika Grabowska.

4. Il est le seul à ne pas faire partie des traducteurs d'auteurs que nous avions contactés auparavant. Cependant, il a le mérite d'avoir traduit certains textes de Ryszard Kapuściński.

5. Cf. aussi les réponses des traducteurs de la littérature polonaise en français dans Skibińska 2012, à paraître.

6. Cf. note 1 à la page 1 .

7. Cf. Cook 2012, à paraître.

\section{AUTEUR}

\section{MONIKA GRABOWSKA}

Monika Grabowska est maître de conférence à l'Institut de Philologie romane de l'Université de Wroclaw. Chef du Laboratoire de Langues romanes, elle s'intéresse à la didactique du FLE, à l'enseignement interculturel, à la politesse linguistique et à la médiation interlinguistique (dont la traduction).

mongr1@poczta.onet.pl 